\title{
Forme des tiges d'érable à sucre et de hêtre à grandes feuilles dans une jeune futaie selon leur position sociale et leur âge
}

\author{
C.-H. Ung
}

Forêts Canada, Centre de Foresterie des Laurentides, 1055, rue du P.E.P.S., C.P. 3800, Sainte-Foy (Québec), GIV 4C7, Canada

(reçu le 29 mars 1988, accepté le 15 décembre 1988)

Résumé - Dans le cadre de l'aménagement intensif d'une jeune futaie expérimentale, nous avons vérifié la pertinence de l'utilisation de tarif́s de cubage séparés selon l'âge et la position sociale des individus à cuber.

Les données disponibles proviennent d'érables à sucre et de hêtres à grandes feuilles abattus dans une jeune futaie d'une quarantaine d'années, située dans la région du Témiscouata. Chaque tige est caractérisée par le coefficient de décroissance, le coefficient d'empattement, l'angle de défilement et le coefficient de forme.

Pour la majorité des arbres de la jeune futaie à l'étude, la position sociale et l'âge de l'arbre (à 50 ans, 40 ans et 30 ans) ne justifient pas l'utilisation de tarifs différents. Néanmoins, la présence du groupe marginal d'érables dominants et de celui constitué presque exclusivement d'arbres âgés de 30 ans, est l'amorce d'une différenciation dans la forme, selon la position sociale et selon l'âge qui se manifestera plus clairement avec le vieillissement du peuplement.

Acer saccharum - Fagus grandifolia - forme de tiges - tarif de cubage - analyse de tiges.

Summary - Stem of sugar maple and beech in a young high forest according to social class and age. In the framework of intensive management of an experimental young high forest, we proposed to examine the exactitude of using volume tables stratified according to the age and the social position of the trees of which the volume was to be estimated.

Data were collected from 37 sugar maples and 40 beeches felled during an experimental thinning. The study was carried out in a mixed hardwood seedling stand in the Témiscouata-Restigouche section (L6, Rowe, 1971), Quebec, Canada. Each stem is characterized by the taper quotient (ratio of dbh to stump diameter), the base quotient (ratio of diameter at mid-height to dbh), the taper angle $\theta=[(\mathrm{dbh}-\mathrm{dm}) /(\mathrm{H}-2.6)]$ where dbh is the diameter of the stem at a height of $1.30 \mathrm{~m}$ (breast height) and $\mathrm{dm}$ is the diameter at mid-height and $\mathrm{H}$ the total height, and by the form factor (ratio of the stem volume to the volume of a cylinder of diameter dbh and height $H_{\text {.) }}$ )

First, the clustering technique is used to detect groups of homogeneous trees in terms of their form; then, a volume table is established for a given group of trees.

For the majority of the trees in the young high forest, the social position and the age of the tree ( 50 years, 40 years, and 30 years) do not justify the use of a stratified volume table. Nevertheless, 
the presence of the marginal group of dominant sugar maple trees and of the marginal group of 30 years old, shows the beginning of a differentiation in the form according to the social position and age. This differentiation will be manifested more clearly with the aging of the stand.

The study sample is small and limited to one stand. However, it offers a contribution to the study of stem form and its impact on tree standing volume estimation.

Acer saccharum - Fagus grandifolia - stem form - volume table - stem analysis

\section{Introduction}

Les forestiers ont, depuis longtemps, reconnu l'importance du défilement du tronc de l'arbre pour faire les estimations précises de son volume. On dit que le tronc défile parce que l'épaisseur décroît sur toute sa longueur pour atteindre à la découpe au fin bout une épaisseur minimale au-delà de laquelle la grume n'est plus utilisable. Ici, il convient de mentionner que le cubage des grumes (Alemdag, 1978; Chaudé et Décesse, 1981) est exclu de notre travail.

Lors des premiers travaux, les dendrométriciens ont été préoccupés par le concept du coefficient de forme, qui permet, à partir du volume d'un cylindre de hauteur $h$ et de diamètre $d$, de calculer le volume du tronc d'un arbre de hauteur $h$ et de diamètre à hauteur de poitrine $d h p$. Ce concept est largement répandu et continue à être utilisé de nos jours (Zarnovican, 1979). Il a l'avantage d'être simple et de permettre de cuber rapidement l'arbre. II est ensuite devenu évident que, malgré son utilité, le coefficient de forme ne pouvait permettre une estimation des proportions du volume total, adaptée au déroulage, au sciage ou simplement au débitage du bois de chauffage.

Depuis les 80 dernières années, de nombreuses études ont été consacrées au choix d'expressions mathématiques appropriées à la représentation du défilement des arbres. Leur forme générale est la suivante :

$$
-\frac{d}{d h p}=f\left(\frac{h}{H}\right)
$$

$d$ étant le diamètre de la tige à la hauteur $h$, dhp le diamètre à hauteur de poitrine et H la hauteur totale de l'arbre. Le problème consiste alors à ajuster les paramètres de la fonction $f$ afin de représenter le plus fidèlement possible, eu égard à un critère donné, l'ensemble des défilements mesurés sur le terrain. La fonction $f$ peut passer d'une forme mathématique simple (Jonson, 1910) à complexe (Hradetzky, 1981 cité par Zarnovican et Ouellet, 1987). La méthode d'estimation des paramètres de la fonction $f$ peut passer de très simple (Jonson, 1910) à sophistiquée (Fries et Matern, 1965; Newberry et Burkhart, 1986). Un grand nombre de travaux se situent entre ces deux extrêmes, comme par exemple, Kozak et al. (1969), Naslund (1980), Palm (1981), Dagnélie et al. (1985).

Mais, sauf de rares exceptions (Chaudé, 1982; M'Hirit et Postaire, 1984), ces modèles sont établis dans le but de décrire le défilement moyen de tous les arbres étudiés. Plus les ensembles d'arbres à 
cuber sont grands, plus les erreurs moyennes sont réduites. Pour des nombres d'arbres plus importants, nous pourrions croire que les erreurs continuent à diminuer indéfiniment. Mais, très rapidement, se pose le problème de l'adéquation des équations aux situations réelles. En effet, si les erreurs aléatoires continuent à se compenser de mieux en mieux, elles ne peuvent compenser les erreurs systématiques liées au fait que l'équation utilisée ne correspond pas de façon suffisamment précise aux conditions du peuplement considéré et, pour le même peuplement, aux arbres d'âges différents ou de positions sociales différentes.

En d'autres termes, la question qui se pose est le choix de l'échantillon représentatif servant à établir l'équation de régression pour un peuplement donné et, éventuellement, pour un groupe d'arbres dans le même peuplement. Cette délicate question est souvent éludée car les forestiers se contentent jusqu'à présent de ramasser un nombre aussi grand que possible d'échantillons, de trouver la forme de la fonction $f$ la plus appropriée en utilisant dhp et $\mathrm{H}$ comme variables explicatives. Or, pour un diamètre et une hauteur donnés, le volume d'une tige est intimement lié à sa forme. Contrairement à la plupart des travaux sur le cubage des arbres qui consistent à fermer les yeux sur la nature multiple et complexe des sources de variation de la forme du tronc (site, génétique et traitement sylvicole), cette étude fait intervenir très simplement la possibilité de discerner la présence ou l'absence de groupes d'arbres ayant des formes typiques. La mise en évidence de ces groupes permettrait d'établir les tarifs de cubage qui leur seraient spécifiques.

Notre étude se propose de :

- vérifier l'influence de la position sociale de l'arbre sur la forme du tronc,
- vérifier la possibilité de changement de la forme du tronc dans le temps. En somme, nous nous proposons de répondre aux questions souvent posées pour l'utilisation du tarif de cubage, c'està-dire l'adaptation du tarif à l'ensemble des arbres du peuplement inventorié et la validité du même tarif pour des inventaires successivement étalés dans le temps.

L'analyse fait appel à une méthode statistique fort simple : la classification automatique, pour répondre à ces questions qui sont absolument cruciales pour le forestier praticien et pour le dendrométricien orienté vers l'aménagement intensif des boisés de surfaces relativement petites. Mais, il convient de souligner que ce n'est pas la classification automatique qui constitue le nœud de la méthode, mais bien la définition des paramètres de la forme du tronc de l'arbre, pour laquelle il n'existe malheureusement pas de règle universelle. Ceci est facilement compréhensible si on reconnaît le caractère relatif de la forme d'un objet.

Les vérifications sur la forme de l'arbre ont pour but pratique d'établir les tarifs de cubage les plus adéquats possibles, par la suppression de l'erreur systématique que peuvent produire les tarifs établis à l'échelle provinciale ou nationale. II nous apparaissait important que ces tarifs soient construits dans le contexte traditionnel, c'est-à-dire que leurs variables explicatives soient facilement et rapidement mesurables à l'aide d'instruments dendrométriques habituels : le diamètre et la hauteur totale. Contrairement à celui de la plupart des tarifs de cubage qui sont construits à l'échelle provinciale ou nationale, avec des nombres fort élevés d'arbres, le cadre de cette étude est très local, soit une jeune futaie d'une superficie de 5,4 ha faisant l'objet de travaux de recherche pour l'aménagement intensif au Témiscouata (Québec). 


\section{Matériel et Méthodes}

Les données disponibles proviennent de 37 érables à sucre (Acer saccharum Marsh) et 40 hêtres à grandes feuilles (Fagus grandifolia Ehrh).

Ces arbres ont été abattus lors d'une éclaircie expérimentale en 1984. Les tiges ont été tronçonnées en billots de $1 \mathrm{~m}$, des rondelles étant prélevées à chaque section en vue de l'analyse de tige. Le choix des arbres échantillonnés s'est effectué sur le terrain de façon à avoir approximativement le même nombre d'arbres par position sociale reconnaissable dans le peuplement étudié, c'est-à-dire les positions dominante, intermédiaire et dominée.

Appelé «massif Lejeune», le peuplement étudié est une jeune futaie de 5,4 ha provenant d'une coupe à blanc effectuée dans les années 30. II est situé à $47^{\circ} 44^{\prime}$ de latitude nord et à $68^{\circ} 34^{\prime}$ de longitude ouest, à $350 \mathrm{~m}$ d'altitude dans le comté de Témiscouata, province de Québec. Le peuplement fait partie du domaine climacique de l'érablière à bouleau jaune de Grandtner (1966) et aussi de la région forestière des Grands Lacs et du Saint-Laurent, section L6-Témiscouata-Restigouche de Rowe (1972).

Le peuplement est constitué de deux essences principales, l'érable à sucre et le hêtre à grandes feuilles représentant respectivement $45,0 \%$ et $24,4 \%$ du nombre de tiges total. Ces deux essences sont accompagnées essentiellement de bouleau jaune (Betula alleghaniensis Britton) (15,7\%).

La démarche suivie dans l'étude est divisée en deux étapes :

- chercher à déceler, par la classification automatique, les groupes d'arbres homogènes du point de vue de leur forme. Cette classification est schématisée par quatre paramètres de forme définis au paragraphe suivant,

- établir le tarif de cubage pour un groupe d'arbres donné. Le tarif peut être représenté par l'intégrale de l'équation de défilement. Comme pour la plupart des tarifs, les valeurs des paramètres de cette équation sont estimées par la méthode des moindres carrés.

\section{Paramètres décrivant la forme de la tige}

Pour caractériser la forme de la tige, comme M'Hirit et Postaire (1984) l'ont fait pour le cèdre du Maroc (Cedrus atlantica Manetti), nous assi- milons la tige à un assemblage de trois troncs de cône de révolution (Fig. 1). Le premier représente' l'empattement de l'arbre, c'est-à-dire la partie située entre le sol et $1,30 \mathrm{~m}$. Le second permet de décrire le tronc entre $1,30 \mathrm{~m}$ et la mi-hauteur. Le troisième représente la moitié supérieure du tronc. Ces troncs de cône permettent de caractériser directement la forme des tiges, à partir des mesures couramment utilisées dans la pratique forestière avec les instruments actuellement disponibles, à savoir :

- le diamètre à la souche: $d s$

- le diamétre à $1,30 \mathrm{~m}: d h p$

- le diamètre à mi-hauteur : $d m$

- la hauteur totale : $\mathrm{H}$

Afin d'éliminer le facteur taille, et dans le but d'assurer la reconstitution de la forme schématique adoptée à partir des paramètres qui la définissent, nous caractérisons chaque tige par les trois paramètres suivants :

- le coefficient de décroissance $\alpha=d m / d h p$

- le coefficient d'empattement $\beta=d h p / d s$

- l'angle de défilement $\theta=\arctan \left[\frac{d h p-d m}{H-2,6}\right]$
(en radians)

Pour nuancer cette schématisation de la tige, nous adjoignons aux trois paramètres le coefficient de forme :

(où $V$ est le volume de la tige). $\gamma=\frac{4 V}{\pi(d h p)^{2} \mathrm{H}}$

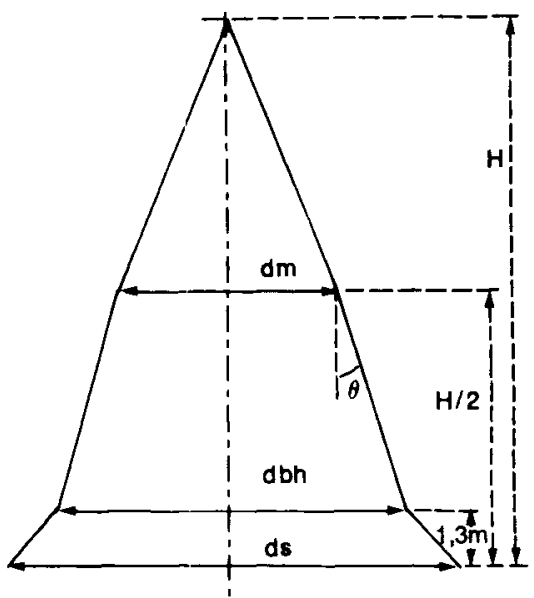

Fig. 1. Schématisation de la forme d'une tige par un assemblage de trois troncs de cône de révolution. ds : diamètre à la souche; chp : diametre à $1,30 \mathrm{~m}$; $\mathrm{dm}$ : diamètre à mi-hauteur; $\mathrm{H}$ : hauteur totale. 
$\mathrm{Ce}$ coefficient donne une indication sur la forme globale de la tige, de la base jusqu'au sommet.

\section{Technique de classification automatique}

La forme de chaque tige, caractérisée par ses quatre paramètres, peut être représentée par un point $X_{i}$ dans un espace quadridimensionnel tel que :

$$
\mathrm{X}_{i}=\left[\begin{array}{l}
\alpha_{i} \\
\beta_{i} \\
\theta_{i} \\
\gamma_{i}
\end{array}\right]
$$

où $i$ varie de 1 à $n$ et $n$ est le nombre d'observations.

Pour la classification automatique utilisée (Batchelor et Wilkins, 1969), la distance entre deux points $X_{i}$ et $X_{i}$ dans l'espace à quatre dimensions est définie par la distance euclidienne:

$$
\mathrm{D}\left(X_{i}, X_{j}\right)=\sqrt{\left(\alpha_{i}-\alpha_{j}\right)^{2}+(\beta-\beta)^{2}+(\theta-\theta)^{2}+\left(\gamma-\gamma^{2}\right.}
$$

Après le calcul des distances euclidiennes entre les points, l'algorithme de classification cherche d'abord les deux points les plus éloi- gnés, qui constituent les centres des deux premiers groupements, affecte ensuite chacun des points qui restent au groupement dont le centre est le plus proche, à condition toutefois pour chaque point que la distance de ce point au centre du groupement ne dépasse pas une certaine fraction de la distance séparant les deux centres initiaux (ici, la moitié). Le cas échéant, si un point est trop éloigné de chacun des deux centres initiaux, il constitue le centre d'un nouveau groupement et la procédure continue.

\section{Tarif de cubage}

II convient de rappeler que notre propos est la recherche de groupes d'arbres ayant une forme moyenne typique. Sachant que la recherche de l'équation la plus appropriée pour le tarif de cubage n'est pas l'objet du travail, nous aurions pu choisir une équation simple, par exemple un polynôme du troisième degré :

Volume total de la tige $=$

$$
a+a b h p)+a(d h p)^{2}+a x(d h p) H+a_{4}\left(d h p^{2} H\right.
$$

avec dhp représentant le diamètre à $1,30 \mathrm{~m}, \mathrm{H}$ la hauteur totale, $a_{0}, a_{1}, a_{2}, a_{3}, a_{4}$, les coefficients de régression (Palm et Rondeux, 1976).

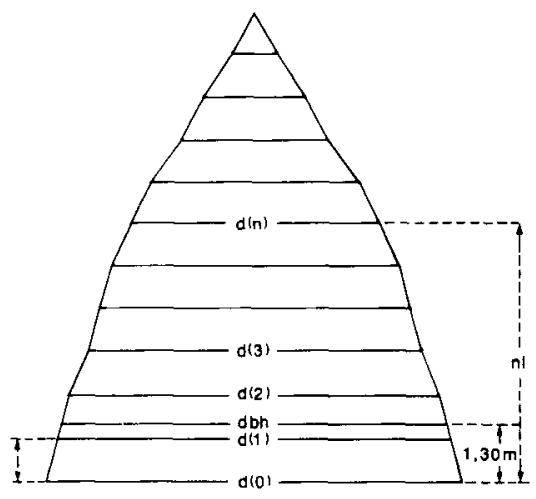

(a)

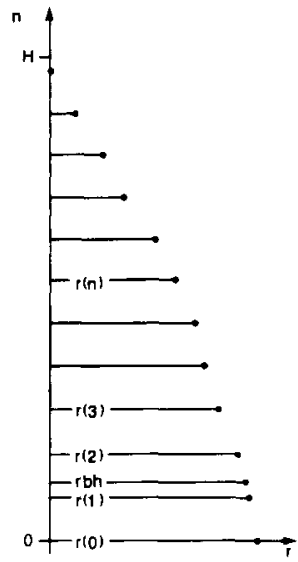

(b)

Fig. 2. Défilement. (a) Segmentation de la tige. (b) Défilement mesuré. $d(n)$ : diamétre à hauteur $n /(n=0,1,2$, $\ldots, \mathrm{L}) ; d h p=$ diamètre à $1,30 \mathrm{~m} ; r(n)$ : rayon à $1,30 \mathrm{~m}$. 
Mais, pour rester dans le cadre du travail sur le défilement, nous avons opté pour une équation représentant le défilement. L'intégrale de cette équation, depuis la base jusqu'au sommet de l'arbre, permet l'obtention du volume total de la tige. En raison de sa simplicité, le modèle choisi est celui développé par Kozac et al. (1969).

$$
x^{2}=b(y-1)+c\left(y^{2}-1\right)
$$

avec $b, c$ : coefficients de régression; $x=\frac{r(n)}{r b h}$; $n=0,1,2, \ldots, \mathrm{L} ; y=\frac{n l}{\mathrm{H}}=\frac{h}{\mathrm{H}} ; n=0,1,2, \ldots, \mathrm{L}$

$x$ étant le rayon réduit, $r(n)$ le rayon de la tige à la hauteur $h=n l(n=0,2, \ldots, L)$, rbh le rayon à $1,30 \mathrm{~m}, y$ la hauteur réduite, $\mathrm{H}$ la hauteur totale de l'arbre.

Pour chaque arbre, la hauteur totale $\mathrm{H}$ est segmentée en $\mathrm{L}$ sections égales de longueur I. L'ensemble des mesures est complété par la détermination du diamètre à $1,30 \mathrm{~m}$. Nous avons alors la segmentation de la tige et le défilement représentés sur les Fig. $2 \mathrm{a}$ et $2 \mathrm{~b}$.

Pour comparer les performances des tarifs spécifiques à celles du tarif général, les critères utilisés sont basés sur les erreurs de cubage résultant de l'emploi des tarifs. Ces critères sont la moyenne de l'erreur absolue et son écarttype, l'erreur absolue étant définie par :

$$
\begin{aligned}
& e=\mid \text { volume observé de l'arbre i } \\
& \text { - volume calculé de l'arbre i } \mid
\end{aligned}
$$

\section{Résultats}

Forme du tronc selon la position sociale

II est bien établi que le rapport $\mathrm{H} / d h p$ reflète la différenciation des arbres dans l'axe vertical du peuplement en classes dominante, intermédiaire et dominée. Zarnovican et Trencia (1987) ont d'ailleurs mis en évidence sur les érables et hêtres de notre étude, l'existence de différentes positions sociales définies par la hauteur totale et le diamètre. En utilisant les équa- tions présentées auparavant, il est possible de vérifier si la considération des positions sociales améliore la précision des tarifs.

D'après les performances des divers tarifs présentés au Tableau I, l'amélioration de la précision apportée par les positions sociales est négligeable. Ce résultat corrobore celui de la classification automatique. Pour l'érable à sucre, la Fig. 3 montre un grand groupe composé de tiges de toutes les positions sociales et un groupe marginal constitué de quatre dominants. Pour le hêtre, la Fig. 4 montre un grand groupe contenant les arbres de toutes les positions sociales, un groupe marginal constitué de deux dominants qui peuvent être qualifiés de gros et trapus et

Tableau 1. Performance du tarif général et des

\begin{tabular}{|c|c|c|c|}
\hline \multicolumn{2}{|c|}{$\begin{array}{l}\text { Performance du tarif } \\
\text { général }\end{array}$} & \multicolumn{2}{|c|}{$\begin{array}{l}\text { Performance des tarifs } \\
\text { spécifiques }\end{array}$} \\
\hline $\begin{array}{l}\text { Erreur } \\
\text { moyenne } \\
\left(d m^{3}\right)\end{array}$ & $\begin{array}{l}\text { Ecart-type } \\
\text { des erreurs }\end{array}$ & $\begin{array}{l}\text { Erreur } \\
\text { moyenne } \\
\left(d m^{3}\right)\end{array}$ & $\begin{array}{l}\text { Ecart-type } \\
\text { des erreurs }\end{array}$ \\
\hline \multicolumn{4}{|c|}{ Erable : 37 arbres } \\
\hline \multicolumn{4}{|c|}{ Position dominante } \\
\hline 1,91 & $\begin{array}{l}\text { Position in } \\
0,90\end{array}$ & $\begin{array}{l}\text { htermédiair } \\
1,74\end{array}$ & 1,65 \\
\hline 0,86 & 0,64 Position & $\begin{array}{l}\text { opprimée } \\
0,73\end{array}$ & 0,62 \\
\hline \multicolumn{4}{|c|}{ Hêtre : 40 arbres } \\
\hline \multicolumn{4}{|c|}{ Position dominante } \\
\hline 3,99 & 3,70 & 3,97 & 3,75 \\
\hline \multicolumn{4}{|c|}{ Position intermédiaire } \\
\hline 1,67 & 1,53 & 1,68 & 1,61 \\
\hline \multicolumn{4}{|c|}{ Position opprimée } \\
\hline 0,86 & 1,57 & 0,80 & 1,31 \\
\hline
\end{tabular}
tarifs spécifiques pour l'érable et le hêtre selon la position sociale. 
4 TIGES

33 TIGES

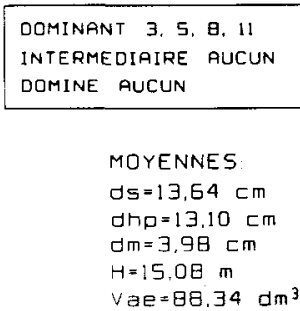

DOMINANT. 1.2. 4, 6, 7, 9, 10
12. $13,14,15,16$

INTERMEDIAIRE 17,19,19,20, 21

22. $23,24,25,26$

DOMINE $27,28,29,30,31,32$.

33. $34,35,36,37$

$$
\begin{aligned}
& \text { MOYENNES } \\
& d s=9.90 \mathrm{~cm} \\
& d h p=8.65 \mathrm{~cm} \\
& d m=2.99 \mathrm{~cm} \\
& H=12.07 \mathrm{~m}^{3} \\
& V a e=44.72 \mathrm{dm}^{3}
\end{aligned}
$$

Fig. 3. Classification des 37 tiges d'érable. ds : diamètre à la souche; $d h p$ : diamètre à $1,30 \mathrm{~m}$; $d \mathrm{~m}$ : diamètre à mihauteur; $\mathrm{H}$ : hauteur totale; Vae : volume avec écorce; $\mathrm{D}$ : distance euclidienne.

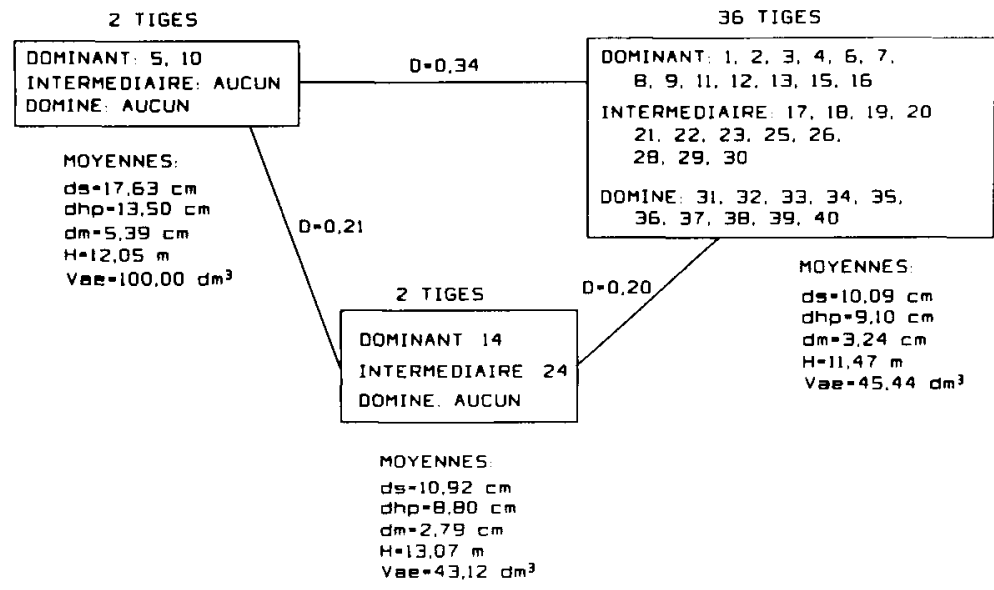

Fig. 4. Classification des 40 tiges de hêtre. $d s$ : diamètre à la souche; $d h p$ : diamètre à 1,30 m; $d m$ : diamètre à mihauteur; $\mathrm{H}$ : hauteur totale; Vae : volume avec ecorce; $\mathrm{D}$ : distance euclidienne.

un autre groupe composé d'un dominant et d'un intermédiaire qui peuvent être qualifiés d'élancés.

La position sociale, discernable par le simple rapport $\mathrm{H} / d \mathrm{~d} p$, n'a pas d'influence sur la forme du tronc examinée sous l'angle de l'établissement des tarifs de cubage pour la jeune futaie et n'apporte pas d'amélioration appréciable à la précision des tarifs de cubage.
Néanmoins, la présence du groupe composé exclusivement de quatre érables dominants nous oblige à nuancer la conclusion sur la position sociale. En effet, ces quatre dominants nous portent à croire qu'ils constituent les premiers représentants auxquels se joindront certains autres dominants dont la forme se distinguera de plus en plus nettement de celles des autres au fur et à mesure que le peuplement vieillira. 
Forme du tronc selon l'âge

II convient de mentionner que la jeune futaie est presque équienne, puisque, pour $92 \%$ des arbres, l'âge varie de 38 à 58 ans. Etant donné que les données proviennent de l'analyse de tige, il nous est possible d'étudier la forme de la tige selon l'âge. Nous aurions pu considérer les 77 tiges ensemble. Mais, afin d'améliorer la netteté du résultat pour le facteur âge, nous avons jugé bon d'analyser un groupe d'arbres plus homogène. Pour ce faire, les 77 tiges ont été soumises à la classification automatique dont le résultat est présenté à la Fig. 5.

Celle-ci montre trois groupes, chaque groupe est composé d'érables et de hêtres. Dans le groupe 1, les arbres ont une forme un peu plus cylindrique que dans le groupe 2. Le groupe 3 est intermédiaire entre les deux premiers. Le groupe 2 étant prédominant a été choisi pour l'étude. Deux groupes de comparaisons ont été effectués, d'une part, les arbres âgés de 50 ans ont été comparés à ceux de 40 et, d'autre part, les arbres de 40 ans ont été comparés à ceux de 30 ans. Le groupe 2 composé de 40 arbres donne lieu à 80 observations dont 40 effectuées sur des arbres de 50 ans et 40 sur des arbres de 40 ou 30 ans.

Pour vingt ans d'intervalle, le résultat de la classification (Fig. 6) présente un grand groupe de 54 observations dont 32 sur des arbres de 50 ans et 22 sur des arbres de 30 ans. Nous ne pouvons pas dire que, pour la jeune futaie, la forme des tiges des arbres de 50 ans est différente de celle des arbres de 30 ans.

Pour l'intervalle de dix ans (Fig. 7), nous constatons un grand groupe de 58 observations dont 33 sur des arbres de 50 ans et 25 sur des arbres de 40 ans. Nous ne pouvons pas dire non plus que, pour la futaie étudiée, la forme des tiges d'arbres de 50 ans est différente de celles d'arbres de 40 ans.

Néanmoins, il convient de souligner (Fig. 6), la présence du groupe de douze observations dont seulement une sur un arbre de 50 ans et onze sur des arbres de

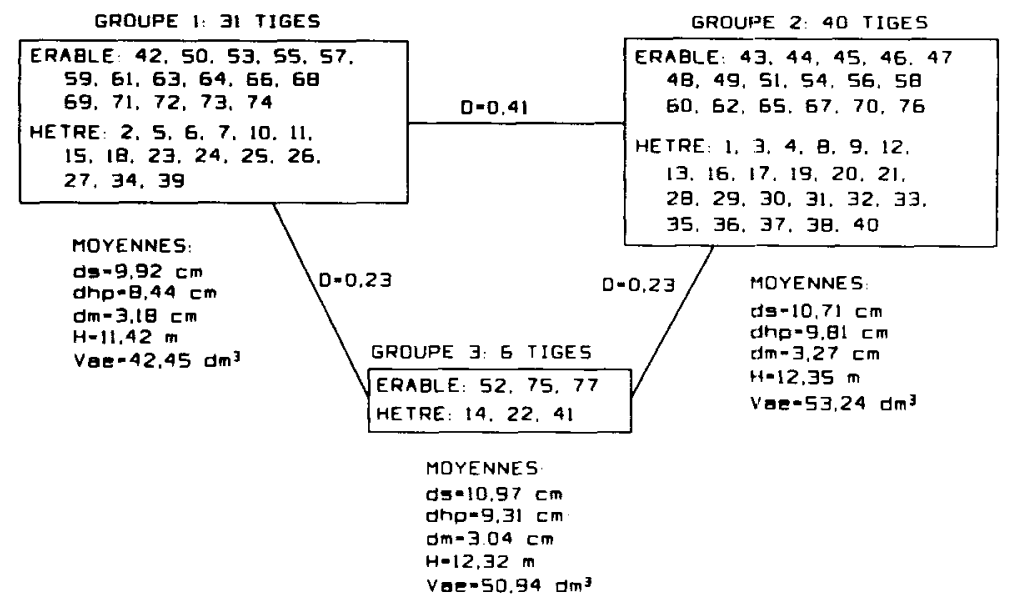

Fig. 5. Classification des 77 tiges d'érable et de hêtre. ds : diamètre à la souche; $d h p$ : diamètre à $1,30 \mathrm{~m} ; d m$ : diamètre a mi-hauteur; $\mathrm{H}$ : hauteur totale; Vae : volume avec écorce; $\mathrm{D}$; distance euclidienne. 


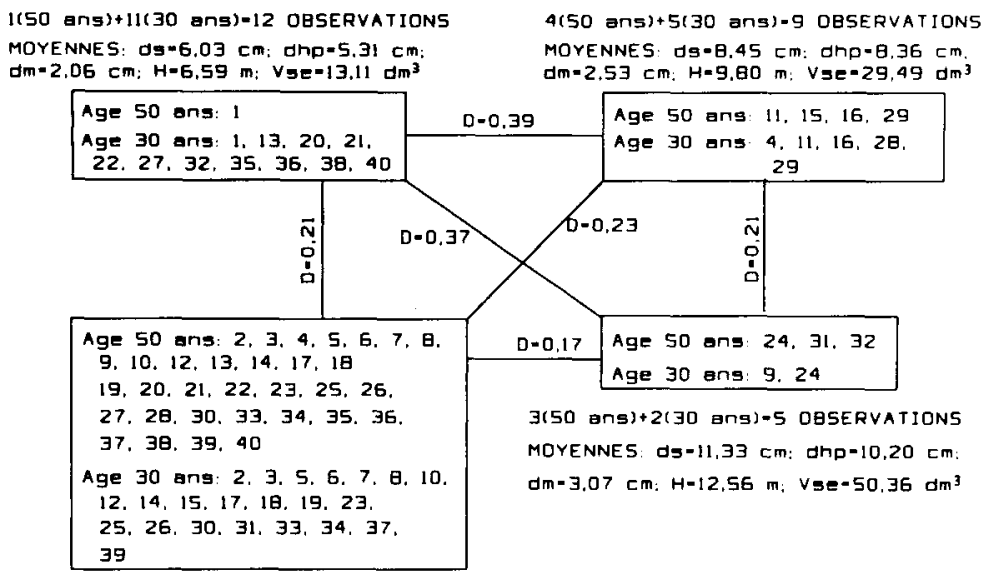

$32(50$ ans) +22(30 ans)-54 OESERVATIONS

MOYENNES d9-8,59 cm; dhp-7.75 cm

$\mathrm{dm}=2.66 \mathrm{~cm}: \mathrm{H}=10.21 \mathrm{~m}: \mathrm{Vse}-31.80 \mathrm{dm}^{3}$

Fig. 6. Classification des 80 observations fournies par les 40 tiges (sans écorce) du groupe 2 mesurées à 50 ans et 30 ans. $d s$ : diamètre à la souche; $d h p$ : diamètre à $1,30 \mathrm{~m}$; dm : diamètre à mi-hauteur; $H$ : hauteur totale; Vse : volume sans écorce; $D$ : distance euclidienne.

30 ans. Les observations effectuées sur ce groupe nous portent à croire qu'avec le temps, c'est-à-dire avec le vieillissement du peuplement, le fossé entre les deux bornes de l'intervalle se creuse, c'est-àdire que la forme du tronc entre les deux bornes se différenciera nettement. Cette constatation contribue à confirmer l'accep-

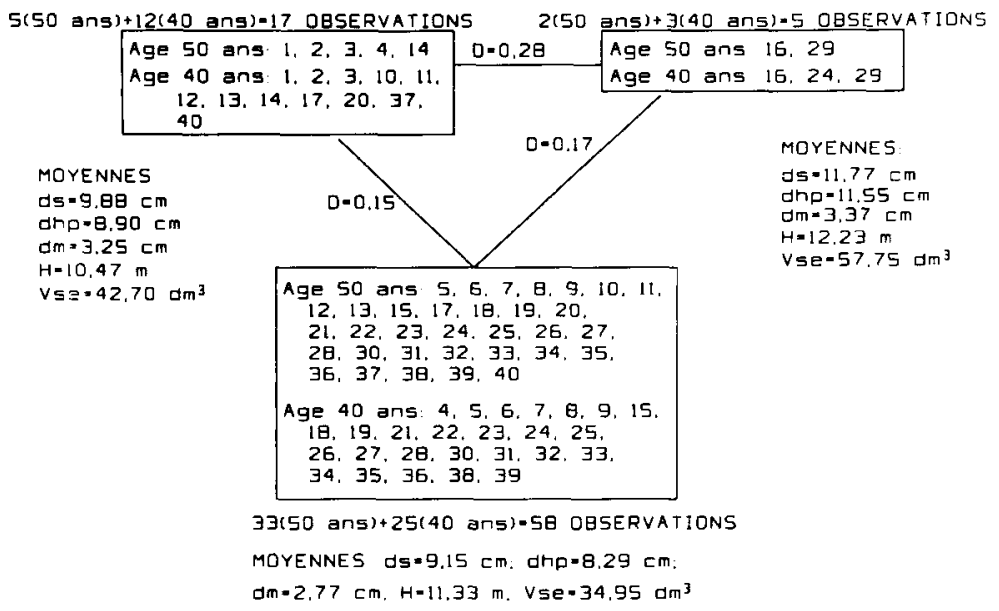

Fig. 7. Classification des 80 observations fournies par les 40 tiges (sans écorce) du groupe 2 mesurées à 50 ans et 40 ans. $d s$ : diamètre à la souche; $d h p$ : diamètre à $1,30 \mathrm{~m}$; $d m$ : diamètre à mi-hauteur; $H$ : hauteur totale; Vse : volume sans ecorce; D : distance euclidienne. 
tation courante que le tarif de cubage établi avec les données prises à un moment donné, ne sera plus valide pour les arbres du même peuplement dans le futur. Malheureusement, nous ne pouvons pas approfondir cette question en augmentant davantage la durée de l'intervalle car l'analyse de tige a été faite sur les rondelles prélevées sur des billots longs de 1 $\mathrm{m}$. Ainsi, nous ne pouvons pas obtenir un modèle du défilement du tronc de l'arbre âgé de 10 ou 20 ans aussi réaliste que celui d'un arbre âgé de 50 ans.

\section{Conclusion}

De la même façon que M'Hirit et Postaire (1984) l'ont fait pour le cèdre du Maroc, nous avons traité le problème de l'estimation du volume des arbres sur pied comme un problème de reconnaissance des formes. En raison de multiples facteurs qui interviennent dans la forme des arbres, le problème est complexe et doit être abordé avec discernement. La caractérisation de la forme du tronc par les quatre paramètres (coefficients de décroissance, d'empattement, de forme et angle de défilement) a permis, dans le cadre de cette étude, de répondre de façon adéquate aux questions que nous nous sommes posées dans le cadre de l'établissement des tarifs de cubage. Pour les érables et les hêtres de la jeune futaie, les résultats de l'étude ont montré une homogénéité relativement grande de leur forme et ont permis de nous rassurer sur le fait que, pour l'estimation du volume sur pied des arbres du peuplement étudié, un seul tarif permet de cuber ces arbres avec une précision acceptable, à l'aide des variables explicatives traditionnelles et rapidement mesurables sur le terrain.

Cependant, la mise en évidence du groupe marginal d'érables dominants et de celui constitué presque exclusivement d'observations effectuées sur des arbres de 30 ans, nous porte à croire qu'une différenciation dans la forme, selon la position sociale et selon le temps, s'amorce dans la jeune futaje étudiée. Du point de vue du tarif de cubage, ces groupes, pour l'instant marginaux, nous poussent à souligner le caractère temporaire du tarif. En effet, construit ì un moment donné, le tarif n'est valide que pour une période donnée dans la vie du peuplement forestier. Sa mise à jour est nécessaire en raison du caractère dynamique de la forme de l'arbre durant sa vie.

\section{Références}

Alemdag I.S. (1978) An analytical look at the log volume formulas. Can. For. Serv., For. Pest Manage. Inst. Sault-Ste-Marie, Ont., 17 pp.

Batchelor B.G. \& B.R. Wilkins (1969) Method for location of clusters of patterns to initialize a learning machine. Electr. Lett. 5(20), 481-483

Chaudé P. (1982) Tarif de Cubage à Décroissances Variables pour les Arbres sur Pied. SOPEDI, France, 49 pp. (+ tableaux)

Chaudé P. \& E. Décesse (1981) Barême de cubage des bois en grume d'aprés leur circonférence médiane. Grumes d'ceuvre, bois d'industrie. Féd. nat. syndicats exploitants for., scieurs et industrieis, $285 \mathrm{pp}$.

Dagnélie P., R. Palm, J. Rondeux \& A. Thill (1985) Tables de cubage des arbres et des peuplements forestiers. Presses Agron. Gembloux, Belgique, $148 \mathrm{pp}$.

Fries J. \& B. Matern (1965) On the use of multivariate methods for the construction of tree taper curves. IUFRO, Royal Coll. For., Stockholm, Res. Note 9, 85-117

Grandtner M.M. (1966) La Végétation Forestière du Québec Méridional. Presses Univ. Laval, Québec, 216 pp.

Hradetzky J. (1981) Spline-Funktionen und ihre Anwendung in der forstlichen Forschung. Forstw. Zbl. 100, 45-59

Jonson T. (1910) Taxatoriska undersokningar of ver skogsträdens form. I. Sven. Skogsvaarsdsfoeren. Tidskr. 8, 284-328 
Kozak A., D.D. Munro \& J.H.G. Smith (1969) Taper functions and their applications in forest inventory. For. Chron. 45, 278-283

M'Hirit O. \& J.G. Postaire (1984) Analyse de la forme des tiges pour la construction des tarifs de cubage. Application au cèdre du Maroc (Cedrus atlantica Manetti). Ann. Sci. For. 4(3), 303-322

Naslund M. (1980) Stem Form Studies of Pine in Northern Sweden. Swedish Univ. of Agric. Sc., Rapp. 8, 86 pp.

Newberry J.D. \& H.E. Burkhart (1986) Variableform stem profile models for lobiolly pine. Can. J. For. Res. 16, 109-114

Palm R. (1981) Contribution méthodologique au cubage des arbres et à la construction de tables de cubage et d'assortiments. Thèse de doctorat, Fac. Sc. Agro., Gembloux, Belgique, $295 \mathrm{pp}$.
Palm R. \& J. Rondeux (1976) A propos de deux problèmes rencontrés dans la construction de tarifs de cubage : le choix des équations de régression et d'échantillonnage des arbres. Bull. Rech. Agron. Gembloux (Belgique) 11, 243-258

Rowe J.S. (1972) Forest Regions of Canada. Dep. Environ. Can. For. Serv. Public. 1300

Zarnovican R. (1979) Comparaison des équations de régression. Application au cubage des arbres d'épinette noire. For. Chron. 55, 54-56

Zarnovican R. \& D. Ouellet (1987) Peuplier faux-tremble au Témiscouata : allométrie, croissance, production et évolution des prix du bois. Serv. can. forêts, Cent. for. Laurentides, Sainte-Foy, Qué., Rap. inf. LAU-X-78, 49 pp.

Zarnovican R. \& J. Trencia (1987) Croissance de l'érable à sucre et du hêtre à grandes feuilles selon la position sociale dans le peuplement. Ann. Sci. For. 44(2), 211-226 